\title{
La céruse dans la fabrication des cosmétiques sous l'Ancien Régime (XVIe-XVIIIe siècles)
}

\section{Catherine Lanoë}

\section{(2) OpenEdition \\ 12 Journals}

Édition électronique

URL : https://journals.openedition.org/tc/224

DOI : $10.4000 /$ tc. 224

ISSN : 1952-420X

Éditeur

Éditions de l'EHESS

\section{Édition imprimée}

Date de publication : 1 mars 2002

ISSN : 0248-6016

\section{Référence électronique}

Catherine Lanoë, « La céruse dans la fabrication des cosmétiques sous l'Ancien Régime (XVIe-XVIIle siècles) », Techniques \& Culture [En ligne], 38|2002, mis en ligne le 11 juillet 2006, consulté le 29

septembre 2022. URL : http://journals.openedition.org/tc/224 ; DOI : https://doi.org/10.4000/tc.224

Ce document a été généré automatiquement le 29 septembre 2022.

Tous droits réservés 


\title{
La céruse dans la fabrication des cosmétiques sous l'Ancien Régime (XVIe-XVIIIe siècles)
}

\author{
Catherine Lanoë
}

De la blancheur du visage sous l'Ancien Régime

1 Travailler sur l'utilisation de la céruse en cosmétique sous l'Ancien Régime nécessite que l'on évoque les exigences de blancheur qui se sont imposées aux hommes et aux femmes de ce temps et que l'on s'interroge sur leur sens ${ }^{1}$.

2 Sous le règne de Catherine de Médicis, le modèle de la société de cour s'installe en France et, avec lui, apparaissent les fards, blanc et rouge. Incontestablement, cette mode est à replacer dans le cadre d'une nouvelle hiérarchie des sens promue par cette nouvelle manière de vivre : la vue s'impose face à l'odorat et au toucher. Dans le cadre de la cour, le blanc du visage est de la même nature que le blanc du vêtement: il témoigne de la distinction de l'aristocrate. Grâce au maquillage, le visage devient donc « un instrument symbolique» (Perrot 1989: 8). Depuis la cour, l'usage des fards se répand parmi l'aristocratie et la bourgeoisie parisienne. Au XVIIIe siècle, à la cour comme à la ville, la blancheur du teint reste une exigence et, bientôt, comme l'a écrit Philippe Perrot (1989 : 48), le maquillage masque les conditions. À ces élites qui veulent conserver des signes distinctifs, les vertus retrouvées du naturel offrent un ultime recours : les fards se font plus discrets; la pâleur reste toutefois ${ }^{2}$.

3 Bien qu'il existe, sans doute, une fabrication artisanale de céruse ${ }^{3}$, sous l'Ancien Régime, la France n'en produit pas en quantité industrielle ${ }^{4}$. Aussi, pour subvenir à ses besoins, elle l'importe de Venise, d'Angleterre et de Hollande. La céruse est vendue chez les apothicaires et/ou les épiciers sous la forme de pains pyramidaux enveloppés de papier bleu, pour en souligner la blancheur ${ }^{5}$; c'est là que particuliers et professionnels de la cosmétique viennent l'acheter. En effet, du XVIe au XVIIIe siècles, la production des cosmé-tiques est double. Il existe, d'une part, une production domestique: des particuliers, suivant les recettes qui figurent dans les manuels, 
élaborent les produits destinés à leur propre consommation. La céruse entre-t-elle dans les préparations que les auteurs de ces manuels offrent au public, et comment? Ce sera l'objet d'un premier axe de réflexion. Il existe, d'autre part, une production professionnalisée : la corporation des parfumeurs, à qui la fabrication de cosmétiques n'appartient pas spécifiquement, souhaite s'emparer du marché. Ont-ils utilisé la céruse ? C'est à cette question que nous consacrerons la deuxième partie. Enfin, nous expliquerons à partir de quand et pourquoi la céruse fut proscrite en cosmétique.

Les manuels de cosmétique

Nous avons concentré notre attention sur quinze manuels de cosmétique publiés entre 1541 à $1782^{6}$. Dans chacun d'entre eux, nous avons mesuré le poids écrasant des recettes pour le teint. Il s'agit de préparations destinées à blanchir le teint, lustrer la peau, ôter le hâle, faire disparaître les taches, les rougeurs, les « rousseurs ", les aspérités de la peau, effacer les rides... Les vertus abondent, semblent souvent interchangeables, quand une préparation ne les réunit pas toutes ${ }^{7}$ ! Pour désigner ces recettes, nous parlerons de préparations blanchissantes parce que, finalement, la blancheur est le but réel. Parmi elles, certaines contiennent de la céruse.

La céruse dans les préparations blanchissantes

5 Avant de préciser de quelle manière la céruse entre dans les préparations cosmétiques, il convient de s'entendre sur le mot: lorsqu'un auteur parle de céruse, s'agit-il toujours de carbonate de plomb? En effet, Jean Liébaut (1582) cite la céruse de froment, de serpentaire (variété d'arum), de corne de cerf, de marbre, de racine, de borax et de camphre. Certaines fois, le mot ne désigne-t-il pas, tout simplement, de la poudre blanche, quelle qu'en soit l'origine ? C'est vraisemblable, car certains auteurs précisent que, pour telle recette, on peut remplacer la céruse par de la poudre d'amidon. Ainsi, André Le Fournier (1541) peut écrire : de la "poudre de fleur de céruse ou amidon autant qu'il suffit pour faire un onguent ». Néanmoins, souvent, les auteurs ont écrit " céruse de Venise ». Pourquoi ? Depuis le Moyen Âge, cette ville est l'un des hauts lieux de la production de céruse en Europe. Aux Temps Modernes encore, la céruse de Venise, rare et très chère, est considérée comme la seule véritable céruse ${ }^{8}$. Lorsque les auteurs précisent "céruse de Venise", ils se fondent sur une "nomenclature d'origine " qui, à elle seule, témoigne de la qualité du produit. La recette qui emploie cette céruse est nécessairement plus efficace.

Voyons maintenant comment la céruse entre dans ces préparations et si nous pouvons en tirer des conclusions. La céruse ne peut être incorporée à une composition cosmétique que sous forme de poudre. Si tel n'est pas le cas, une première étape consiste à la broyer. Michel Nostradamus recommande de la donner à un peintre qui la broiera sur son marbre (1572). La poudre doit être fine, sans aucune aspérité, douce au toucher et très blanche, c'est sa principale qualité. Lémery (1748) propose d'ailleurs l'étymologie du mot : il viendrait de cera, "cire", dont la céruse a la blancheur et la douceur ${ }^{10}$. D'après les manuels du XVIe et du début du XVIIe siècles, une seconde opération consiste à laver la céruse, parfois plusieurs fois, avec des eaux parfumées, et, le plus souvent, avec de l'eau de rose. Est-ce pour en faciliter le broyage si elle se présente en morceaux? On conseille de le faire même s'il s'agit de céruse en poudre. Croit-on atténuer ainsi son caractère corrosif ? C'est possible. Plus vraisemblablement toutefois, il s'agit de la parfumer. La quantité de céruse préconisée semble très variable. Chez André Le Fournier, elle n'est pas toujours mentionnée : dans telle préparation, il écrit 
« un peu de céruse lavée à votre discrétion » et, plus loin, « céruse autant qu'il suffit »" Dans les manuels qui succèdent au sien, les proportions sont toujours indiquées et varient entre une drachme et six onces pour les préparations classiques ${ }^{12}$, soit de un à quarante-huit! Rien ne permet d'expliquer le choix de tel ou tel poids, car nous n'avons pu établir de lien entre la quantité de céruse et le but de la préparation. D'ailleurs, dans un même ouvrage, alternent des préparations à objectif identique, les unes contenant de la céruse et les autres non! Dans la section sur les blancs et magistères cosmétiques de son manuel, Nicolas de Blégny (1689) donne une recette de blanc de céruse mais aussi des recettes de poudre de talc: de manière indifférente pour l'auteur, elles peuvent toutes être mélangées à des pommades.

7 Comment et à quoi la céruse est-elle associée ? Aucune règle ne semble s'imposer, en particulier pour les recettes les plus anciennes, mais la complexité des préparations est toujours de rigueur. Réduite en poudre, la céruse est mêlée à des produits végétaux, eaux de fleurs, jus de fruits, vinaigres, huiles, gommes... et à des produits d'origine animale, graisses en tous genres, œufs, cire... Souvent, elle est aussi associée à d'autres produits minéraux ou métalliques: vif-argent, sel de tartre, alun, soufre, camphre, litharge d'or et d'argent... mais, là encore, il semble difficile de tirer des conclusions. Peut-être, toutefois, pouvons-nous avancer qu'avec le temps, le nombre d'ingré-dients diminue... ${ }^{13}$

8 Comme nous l'avons suggéré, la céruse est l'un des ingrédients de préparations très diverses: des onguents, des pommades, des eaux, des mouchoirs cosmétiques... chacune s'accompagnant, naturellement, de gestes spécifiques. Finalement, c'est bien dans la nature des préparations qu'apparaissent les distinctions diachroniques les plus nettes. Dans les manuels des XVIe et XVIIe siècles, nous avons souvent rencontré le mot "frotter ", presque toujours attaché à la manière d'appliquer les produits contre les taches, les rousseurs, et contre le hâle. Dans les manuels du XVIIIe siècle, les recettes visant à faire disparaître les macules se font moins nombreuses. Aux XVIe et XVIIe siècles, les onguents sont les plus nombreux. Épais et gras, on conseille de les appliquer le soir et de les laisser au contact de la peau un temps assez long: Le Fournier (1541), repris par Liébaut (1582), dit d'une de ses préparations qu'il faut « l'appliquer comme si c'était un faux visage et le tenir toute la nuit ». Au XVIIIe siècle, les préparations ont, plus souvent, une forme aqueuse, et le vocabulaire s'en trouve modifié: le verbe «laver " s'impose, et l'on devine que la durée du contact est beaucoup plus courte. Si les auteurs disent encore qu'il faut appliquer leur produit le soir, l'utilisation du matin est de plus en plus recommandée. D'une certaine façon, cela témoigne des nouveaux gestes de toilette qui se développent parmi les élites au cours du siècle des Lumières et dans lesquels l'eau, fût-elle composée, prend une place inédite ${ }^{14}$.

La céruse dans la préparation des fards

9 Dès l'Antiquité, la céruse a servi à fabriquer du fard blanc : à Athènes et à Rome, les témoignages sur le blanc de céruse abondent ${ }^{15}$. De même, à l'époque moderne, la céruse est employée à cet effet. Régnier écrit : « [...] leur visage reluit de céruse et d'épeautre »; Boileau : [...] la coquette [...] mettant la céruse et le plâtre en usage, composa de sa main les fleurs de son visage ${ }^{16}$. Enfin, La Bruyère déclare que les femmes s'enlaidissent en se fardant de blanc et de rouge et que les hommes « haïssent autant de les voir avec de la céruse sur le visage » (Les Caractères, 1691). La céruse sert ici, comme en peinture, de pigment blanc que l'on mélange à différents corps selon la consistance 
souhaitée : eaux ou huiles parfumées -dans lesquelles elle est délayée-, pommades et blanc de baleine.

Or, dans les manuels de cosmétique, nous n'avons trouvé aucune recette de blanc de céruse qui porte le nom de fard avec l'acception que nous lui connaissons aujourd'hui. Cette constatation nous étonne, mais nous avons une réponse. L'auteur de préparations chimiques compliquées ne daigne pas donner une recette simple, à la portée de tous, car quiconque dispose de céruse peut fabriquer $d u$ fard. Une difficulté surgit pourtant : quelques préparations blanchissantes sont parfois aussi appelées fards. André Le Fournier intitule l'une de ses préparations « une manière de se farder le visage la nuit [...]», et l'on trouve chez Michel Nostradamus un «fard pour enblanchiment de la face », et une « eau [...] s'en fait un fard [...]». Comment interpréter ce terme ? Il y a incontestablement une confusion des mots et des usages: certaines préparations blanchissantes que nous avons décrites sont aussi des fards dont l'auteur tait le nom à dessein. Et comment s'en étonner dans un monde où la beauté naturelle reste la référence, où l'artifice et les fards en particulier sont condamnés, dans un monde où le mot «fard », lui-même, est employé dans le sens de feinte ou de dissimulation (Dubois \& Lagane 1971).

Pourquoi la céruse?

11 La céruse est utilisée en cosmétique car les auteurs des manuels sont souvent des médecins ${ }^{17}$. D'une part, elle leur semble ne pas avoir les effets nocifs des préparations dénoncées dès le XVIe siècle, telles, en particulier, les recettes contenant du mercure ${ }^{18}$. D'autre part, elle profite sans doute de la disqualification de la pharmacie galénique et du développement de la pharmacie chimique. Enfin, elle bénéficie d'un prolongement de ses vertus médicinales déjà reconnues par Pline l'Ancien. Astringente et cicatrisante, elle est aussi capable de "polir, de nettoyer et de blanchir la face », comme l'écrit Liébaut (1582). De plus, les onguents blanchissants sont apparentés de manière évidente aux emplâtres médicaux. Liébaut donne même une recette cosmétique avec du sel de céruse (ou sel de Saturne), et précise qu'il faut l'employer « comme quand on fait des cautères». Les médecins des Temps Modernes qui ont écrit des ouvrages de cosmétique ont considéré que santé et beauté étaient équivalentes.

Finalement, on peut conclure que la céruse est choisie pour trois raisons. Parce qu'elle est corrosive, dessicative, astringente, et que l'on souhaite ôter du visage toute aspérité et toute tache. Parce qu'elle est un pigment blanc dont on peut faire du fard. Ce sont là des propriétés reconnues, objectives. Mais on l'emploie tout autant, voire davantage, parce qu'elle est blanche et que l'on souhaite obtenir la blancheur, socialememt valorisée, au même titre que l'on préfère les pigeons blancs, le pain blanc, le fromage blanc, les fleurs blanches... et pour la même raison que le linge dont on s'essuie le visage au matin doit être blanc. C'est le principe, admis par les médecins paracelsiens, selon lequel «les semblables attirent les semblables» (Metzger 1969: 155) ${ }^{19}$. On saisit ici la valeur subjective, magique de la céruse.

La céruse des parfumeursBoutique et «blancs » de parfumeurs

13 Nous l'avons dit, l'usage des fards blancs ne semble pas reculer au cours des Temps Modernes, comme en témoignent, pour les critiquer d'ailleurs, de nombreux observateurs. Les parfumeurs ont assuré une partie de la production des blancs ${ }^{20}$. Dans son Dictionnaire..., l'Abbé Jaubert (1773) écrit que les parfumeurs fabriquent « un grand nombre de substances pour blanchir et nettoyer qui sont sujettes aux changements car 
il faut toujours en imaginer de nouvelles pour suivre la mode ». En effet, le fonds de boutique des parfumeurs contient des préparations blanchissantes et des fards ${ }^{21}$. Les laits virginaux sont les préparations destinées à blanchir la peau les plus communes: nous en avons trouvés dans treize inventaires après décès mais aussi dans deux registres de commerce ${ }^{22}$. Les eaux sans pareilles (onze inventaire après décès) et les eaux pour le teint (quatre inventaires après décès, trois registres de commerce) font également partie de ces préparations blanchissantes. Les traces archivistiques de fards blancs sont plus rares: «blanc de perles ${ }^{23}$ " cinq fois, «blanc glacé» une seule fois, blanc en pot -sans plus de précision-, trois fois. Selon Fitelieu, il faut disposer d'une boutique entière pour se farder: "tant de céruse, de sublimé, de rouge d'Espagne, d'alun, de mie de pain, de vinaigre distillé, de fèves, de fiente de bœuf, d'amandes... et tout cela ne sert que pour masquer une femme " (Fitelieu 1642). Avec quelles matières premières les parfumeurs ont-ils fabriqué leurs préparations blanchissantes et leurs fards ? Ont-ils utilisé la céruse?

Peu ou pas de céruse chez les parfumeurs

14 L'étude des inventaires après décès a conduit à une première constatation: les parfumeurs ne disposent d'aucun manuel de cosmétique dans leur bibliothèque quand ils en ont une. Leurs savoir-faire se sont transmis de manière orale. Sous l'Ancien Régime, deux mondes cosmétiques ont bel et bien coexisté, sans s'influencer l'un l'autre, semble-t-il.

Bien que les parfumeur utilisent la céruse pour la teinture des gants, ils l'emploient très peu dans les préparations cosmétiques. Simon Barbe (1689), seul parfumeur à avoir écrit un manuel, cite la céruse pour teindre les peaux en blanc ou pour adoucir d'autres couleurs, mais aucune de ses préparations n'en contient. En effet, nous avons trouvé seulement deux inventaires après décès dans lesquels le notaire mentionne du blanc que l'on peut identifier à de la céruse. Dans l'inventaire après décès de Barthélemy Dupré, en 1738, on peut lire: «deux onces de blanc trois livres, six douzaines de pommade au teint 15 livres, lait virginal une chopine 30 sols... » ${ }^{24}$. Dans celui de Philippe Deldeuil, en 1767, le greffier a noté : « item de l'eau sans pareille, quatre onces de pain de blanc à 20 sols l'once »" Pourquoi penser qu'il s'agit de céruse alors que le notaire ne le précise pas? Parce qu'il est difficile de différencier la céruse des autres types de blancs, le terme «blanc » est devenu générique. Surtout, la présentation sous forme de «pain ", ainsi que les quantités, laissent penser qu'il s'agit bien de céruse. De manière plus générale, pourquoi les traces de céruse sont-elles si peu nombreuses? Deux hypothèses se présentent : soit les parfumeurs l'achètent en petite quantité et la transforment tout de suite de peur qu'elle ne s'abîme et perde sa blancheur; en effet, la céruse perd vite sa qualité essentielle : Pons Augustin Alletz (1776) recommande de lui ajouter une pointe de bleu « afin de soutenir le blanc qui jaunit avec le temps »; soit les parfumeurs en connaissent la toxicité -ce n'est pas exclu-, mais ils emploient pourtant d'autres produits dangereux... Dans le fond, la réponse la plus acceptable est peut-être la suivante : ils utilisent peu la céruse parce qu'elle est très chère.

Les matières premières des parfumeurs

16 À la lecture de nos sources, les préparations blanchissantes et les fards des parfumeurs semblent avoir été fabriqués à partir de matières premières nombreuses et variées. Du blanc, sans plus de précision, est mentionné cinq fois, du talc quatre fois, du tartre trois fois, de l'étain une seule fois. La matière première la plus communément employée par 
les parfumeurs est l'alun: nous en avons relevé 18 occurrences! Effectivement, l'Encyclopédie nous confirme que l'alun entre dans les préparations astringentes et dans plusieurs cosmétiques. Dans les inventaires après décès et les registres de commerce que nous avons consultés, cet alun se présente de manières différentes : six fois, aucune précision n'est donnée, une fois il est dit "glacé», trois fois il se présente "en poudre ", et huit fois il est "calciné ». Ces deux derniers types nous intéressent particulièrement et nous pensons qu'il s'agit d'une seule et même forme car, selon l'Encyclopédie: «[...] l'alun calciné sur le feu [...] devient plus blanc, plus léger et plus facile à pulvériser et caustique ». Comme la céruse, l'alun se transforme en poudre blanche; comme elle, il a des vertus astringentes et corrosives.

Pourquoi l'alun plutôt que la céruse ? C'est un produit dont les parfumeurs connaissent les usages et qui, sans doute, coûte moins cher ${ }^{26}$. Enfin, on peut penser que leur qualité de poudriers les a aussi incités à fabriquer leurs fards avec de la poudre d'amidon. Les parfumeurs du XVIIIe siècle ont donc produit et vendu des blancs de toutes sortes: des préparations caustiques avec de l'alun, de la céruse et bien d'autres métaux et minéraux encore, mais aussi des compositions plus ou moins inoffensives, faites de talc ou d'amidon. Et d'ailleurs, d'après ce que nous savons de la consommation, les mêmes clients ont pu avoir recours à différents types de blancs, en alternance ou en les mélangeant. Les parfumeurs ont sans doute souhaité s'emparer d'un marché en développement ; mais en proposant d'autres produits au public, ils ont aussi contribué à la disqualification de la céruse.

La disparition progressive de la céruseUn produit toxique longtemps en usage

Certains auteurs connaissaient les effets nocifs de la céruse depuis le XVIe siècle. En 1582, par exemple, Jean Liébaut en vante les vertus mais dit de la céruse, comme des autres « [...] préparations métalliques [...], qu'elles noircissent et corrodent les dents et rendent l'haleine puante ». Dans son Histoire générale des drogues de 1694, l'épicierdroguiste Pierre Pomet considère que la céruse est une drogue « très dangereuse tant à broyer qu'à mettre en poudre ", il évoque des maladies, parfois la mort, et ne fait pas même mention de son usage en cosmétique. En 1748, Jacques Savary des Brûlons précise que la céruse est « un poison dangereux quand elle opère au-dedans et elle fait même sentir au-dehors sa malignité puisqu'elle gâte la vue et les dents des personnes qui prétendent s'en embellir et qu'outre quantité d'autres incommodités qu'elle leur cause, elle semble avancer la vieillesse, en faisant venir des rides plus tôt qu'on en aurait ». Il est donc légitime de se demander pourquoi la céruse a disparu si lentement des manuels de cosmétique. Plusieurs réponses sont possibles.

Nous l'avons dit plus haut, dans le domaine cosmétique, elle a longtemps bénéficié des vertus curatives qu'on lui reconnaissait. Et l'on peut donc penser que les premières condamnations ont seulement visé la céruse coupée de craie -venue d'Angleterre et de Hollande-, attribuant à la craie les effets nocifs, à tort bien sûr. En effet, Pomet (1694) précise bien qu'en médecine, on doit employer la céruse de Venise et qu'il faut l'acheter chez «d'honnêtes marchands ». De manière plus générale, le succès des manuels de cosmétique témoigne d'une demande : les exigences de blancheur touchent toujours une certaine frange de la population sous l'Ancien Régime. Les auteurs répondent à cette demande, recopient les uns sur les autres des recettes, parmi lesquelles certaines sont absolument inoffensives et d'autres contiennent de la céruse: en soi, l'accumulation des préparations les plus diverses témoigne de la qualité de l'ouvrage qui les propose. Citons tout de même le cas de Mademoiselle Meurdrac qui fait preuve 
d'un peu de lucidité face aux dangers des préparations métalliques: dans l'édition de 1666 de sa Chimie charitable en faveur des dames, elle conseille de ne pas se servir du mercure, du sublimé ou d'étain de glace, et donne quatre recettes qui accueillent de la céruse. La réédition augmentée de son ouvrage (1674) compte davantage de préparations, mais une composition «contre les rides et les lentilles du visage » qui contenait de la céruse a disparu. La demoiselle a-t-elle supprimé cette recette parce qu'elle ne permettait pas d'atteindre l'objectif annoncé ou parce qu'elle avait des effets nocifs? Quelle que soit la réponse, Mademoiselle Meurdrac a fait appel à un raisonnement fondé sur la comparaison entre un objectif et un résultat -et c'est tout à son honneur.

Les «maladies de la céruse »

20 La définition des risques liés à la céruse a été lente car, pendant longtemps, le discours moralisant sur les fards a sans doute occulté toute autre considération, et les informations médicales circulaient peu. Finalement, il faut attendre que l'emploi du plomb soit condamné en médecine pour que l'usage de la céruse soit remis en cause en cosmétique, c'est-à-dire la seconde moitié du XVIIIe siècle. En 1760, le Docteur Maurice Deshais-Gendron s'inquiète de certaines maladies des yeux dont souffrent les femmes qui utilisent les fards ${ }^{27}$. Il parle aussi de salivation, de sécheresse de la bouche, d'inflammation des gencives. Enfin, il évoque, parmi les classes privilégiées, des maladies de poitrine, des affections des poumons qui pourraient venir de l'application de ces blancs sur une plus grande surface du corps. Sa dénonciation des cosmétiques est sans appel : «Vous ne trouverez dans leur composition que poisons, que corrosifs, que dessicatifs, qu'astringents». Le docteur Lorry (1777: 196) est plus précis: il décrit clairement les dangers des préparations au plomb. La blancheur et l'éclat ne séduisent qu'un temps : une utilisation prolongée provoque des taches, la peau devient froide, aride, « des douleurs aiguës et des convulsions effroyables » s'ensuivent. Le médecin raconte qu'une femme qui appliquait du blanc sur une grande partie de son corps depuis trois ans est morte phtisique très rapidement.

21 Si les deux médecins semblent d'accord pour attribuer au fard, et au blanc de plomb en particulier, des effets nocifs, les maux qu'ils décrivent ne sont pas identiques. Surtout, leurs analyses sur la manière dont le plomb s'insinue dans le corps divergent. Pour M. Deshais-Gendron, le fait que l'on applique ces produits au pinceau « ouvre les pores de la peau, excite la dilatation des vaisseaux ce qui laisse entrer les molécules ». Pour M. Lorry, au contraire, le caractère astringent des substances métalliques rétrécit le diamètre des pores; le fard empêche l'indispensable transpiration et, finalement, les molécules métalliques s'insinuent dans le corps ${ }^{28}$. Surtout, le docteur Lorry observe une « analogie [...] entre ces symptômes et les phénomènes des maladies des ouvriers qui travaillent aux mines et aux métaux ». Il évoque des migraines, des lassitudes, des fluxions, des maux d'estomac et de reins... Pouvons-nous effectivement croire qu'il s'agit des symptômes caractéristiques des "coliques de plomb" ou "coliques de peintres»? Peu importe. Deux points sont à retenir : ces médecins présentent des raisonnements fondés sur l'observation et la comparaison de signes cliniques, ce qui témoigne des progrès de la médecine; aux yeux de leurs contemporains, leurs conclusions sont vraisemblables.

La condamnation de la céruse

22 En effet, c'est seulement à partir de la seconde moitié du Siècle des Lumières que la céruse en cosmétique recule de manière significative. Dans l'ouvrage de Le Fournier 
paru en 1541, $28 \%$ des préparations décrites contiennent de la céruse, on en compte $15 \%$ dans le manuel de Liebaut en 1582, $6 \%$ dans celui de Blégny en 1689, $20 \%$ chez Lémery en 1695, 2,8 \% chez Bruzen de la Martinière en 1759, 4 \% chez Buchoz en 1771, pour finir par 2,7\% dans l'ouvrage de Dejean en 1777.

À partir des années 1770, les effets toxiques de la céruse sont reconnus de tous. L'Abbé Jaubert (1773) dresse un véritable réquisitoire contre les préparations qui en contiennent. Les femmes doivent se méfier, car ces préparations "vénéneuses" causent des «mortifications » (sic), altèrent, gâtent, « rident le teint de manière à ne jamais pouvoir le réparer » et font disparaître « ses couleurs naturelles ». Le discours est négatif, il est, terme à terme, l'exact contre-pied de celui qui, deux siècles plus tôt, accompagnait certaines préparations contenant de la céruse. Enfin, Jaubert étend cette condamnation à ceux qui pensent « pouvoir trouver des cosmétiques propres à ôter les taches, les rousseurs, rougeurs, les rides ou changer la couleur de la peau » : ce sont des charlatans.

Surtout, les informations scientifiques et médicales circulent. Les recettes cosmétiques qui sont envoyées par des particuliers à la Société Royale de Médecine pour obtenir une autorisation de vente en témoignent: presque tous ces inventeurs déclarent que leur produit est "sans fard", donc sans danger. La dame Colson écrit que sa crème à la sultane ne "contient point de blanc qui puisse par son usage brunir la peau», Mademoiselle Guérin précise que son eau est exempte de fard et de tout ce qui peut dessécher $^{29}$. Le Sieur de la Houssaie va plus loin encore: il prétend que son eau spiritueuse est un "véritable contrepoint du rouge et du blanc qui gâtent le teint des personnes qui en font usage ${ }^{30}$. Certains font preuve de connaissances chimiques très sûres : le sieur Viserant envoie la recette d'un vinaigre cosmétique et précise qu'il faut éviter, pour le préparer «[...] de choisir un vaisseau de terre couverte d'un émail de plomb car il peut se former par l'acide acéteux qui tombe dessus un sel de saturne [...] », ou sel de céruse, que l'auteur qualifie "de poison dangereux » ${ }^{31}$. L'affirmation selon laquelle la préparation ne contient « aucun fard » revient comme un leitmotiv, et, finalement, elle devient l'un des arguments majeurs en faveur de la recette, pour ne pas dire l'argument publicitaire principal. C'est aussi celui que la communauté des parfumeurs, inquiète de perdre le marché des cosmétiques face à cette nouvelle concurrence, avance: la disparition des corporations donne lieu à une production spontanée et potentiellement dangereuse pour le public; il conviendrait, donc, de les établir, à l'exclusion des autres, seuls fabricants de cosmétiques ${ }^{32}$.

En revanche, lorsque la Société Royale reçoit des préparations douteuses et dangereuses, le jugement est sans appel. Nous n'avons retrouvé aucune recette qui contienne directement de la céruse; mais nous en avons découvert deux avec du sel de saturne que l'on fabriquait avec de la céruse ${ }^{33}$. À l'examen de l'un de ces cosmétiques, le jugement de la Société est le suivant :

«Il se forme un vinaigre de saturne duquel par un mélange d'eau distillée, il se précipite une poudre blanche qui reste au fond des vases. Dans cette poudre, on revivifie le plomb par les moyens les plus simples [...] [et elle] [...] peut être regardée comme un blanc de céruse [...] qui donne nécessairement à l'eau une qualité astringente et une vertu [illisible] dont l'action est justement suspecte dans les maladies cutanées ${ }^{34}$.

Conclusion 
Même si la condamnation des fards par les médecins du XVIIIe siècle com-porte incontestablement une dimension morale, ces hommes ont le mérite d'avoir fait admettre que, si médecine et cosmétique ont en commun le do-maine de la peau, elles n'ont pas la même fonction. Plus important encore : comme la recherche de la beauté peut être nuisible, le corps médical souhaite contrôler la production des cosmétiques. Ces médecins avaient raison, et ils ont sans doute largement concouru à l'élimination de la céruse des prépa-rations cosmétiques au XVIIIe siècle. Mais la victoire de la raison n'est pas toujours définitive... Deux siècles plus tard, on sait que, dans plusieurs pays et en particulier sur le continent africain, circulent des produits blanchissants dangereux ou que certains médicaments sont utilisés de manière détournée pour blanchir la peau, avec tous les effets secondaires désastreux que l'on imagine ${ }^{35}$. Là encore, il apparaît que la blancheur de la peau fait partie d'un ensemble de valeurs qui appartiennent au modèle économique et social dominant. Comme elle s'était imposée aux classes populaires sous l'Ancien Régime, elle semble parfois s'imposer aujourd'hui aux peuples non européens.

\section{BIBLIOGRAPHIE}

Alletz Pons, Augustin

1776 Albert moderne ou nouveaux secrets éprouvés et licites...

Bachelard, Gaston

1938 La Formation de l'esprit scientifique. Paris : Vrin.

Bénézet, Jean-Pierre

1999 Pharmacie et médicament en Méditerranée occidentale, XIIIe-XVIe siècles. Paris : Champion.

Barbe, Simon

1689 Le Parfumeur royal. Paris.

Blégny, Nicolas (de)

1689 Secrets concernant la beauté et la santé, tome 2. Paris

Bruzen de la Martinière

1759 Art de conserver la santé... augmenté d'un traité sur la conservation de la beauté des dames. Paris.

Buchoz, Pierre-Joseph

1771 Toilette de Flore.

Dejean

1777 Traité des odeurs. Paris.

Dubois, J., R. Lagane \& A. Lerond

1971 art. « fard », p. 253, in Dictionnaire du Français Classique.

Elias, Norbert

1985 La Société de Cour. Paris : Flammarion. 
1991 La Civilisation des mœurs. Paris :Presses Pocket.

Fitelieu

1642 La Contre mode. Paris.

Garçon, Anne-Françoise

1998 Mine et métal. Rennes : Presses Universitaires de Rennes

Girre, Loïc

1997 Traditions et propriétés des plantes médicinales. Toulouse : Privat.

Goncourt (de), Edmond et Jules

1887 La Femme au XVIIIe siècle. Paris

Goubert, Jean-Pierre

1986 La Conquête de l'eau. Paris : Robert Laffont.

Jaubert (Abbé)

1773 Dictionnaire raisonné universel des arts et métiers... Paris.

Kaplan, Steven L.

2001 La Fin des corporations. Paris : Fayard.

Le Breton, David

1992 Des visages. Paris : Métailié

Le Chevalier Digby

1689 Remèdes souverains et secrets. Paris.

Le Fournier, André

1541 La Décoration d'humaine nature. Paris.

Lémery, Nicolas

1695 Recueil des plus beaux secrets...

1748 Dictionnaire universel de médecine (édition révisée de l'ouvrage de Pierre Pomet, Paris 1694).

Jean Liébaut

1582 Trois livres de l'embellissement du corps humain. Paris.

Lorry (Dr)

1777 « Observations sur la manie de blanchir la peau avec des préparations métalliques et sur les accidents qui en résultent », Le Journal de médecine 48.

Mandrou, Robert

1989 Introduction à la France moderne. Paris : Albin Michel.

Mauss, Marcel

1902-03 Esquisse d'une théorie générale de la magie. Paris : Année sociologique.

Metzger, Hélène

1969 Les Doctrines chimiques en France du début du XVIIe à la fin du XVIIIe siècle. Paris : Albert Blanchard (1ère édition 1923).

Mademoiselle Meurdrac

1674 Chimie charitable en faveur des dames. (1ère édition 1666).

Nostradamus, Michel

1572 Excellent et très utile opuscule à tous nécessaire, de plusieurs exquises recettes... Lyon. 
Perrot, Philippe

1984 Le Corps féminin XVIIIe-XIXe siècles. Paris : Seuil.

Pline l'Ancien

Histoire Naturelle, XXXIV, Sur le plomb. Les Belles Lettres.

Pomet, Pierre

1694 Histoire générale des drogues. Paris.

Savary, Jacques

1748 Dictionnaire universel de commerce. Paris.

Vigarello, Georges

1985 Le Propre et le sale. Paris : Seuil.

\section{NOTES}

1. Ces lignes doivent beaucoup à plusieurs ouvrages; citons les plus importants pour notre propos. Les analyses de Norbert Elias $(1985,1991)$ se sont avérées essentielles pour comprendre les liens entre l'espace curial et le développement des soins du corps. Les belles pages de Robert Mandrou (1989) sur les modifications de la hiérarchie des sens au début des Temps Modernes nous ont été très utiles. Le livre de Philippe Perrot (1984), et celui de Georges Vigarello (1985) sont indispensables pour qui souhaite aborder la question des soins du corps et de beauté dans la France d'avant 1789. Enfin, l'ouvrage de l'anthropologue David Le Breton (1992) permet d'éclairer le sujet d'une manière fort intéressante.

2. Selon Edmond et Jules de Goncourt (1887), à la fin du XVIIIe siècle, les coiffures s'avancent sur le front pour faire de l'ombre au visage et, ainsi, le faire paraitre plus blanc. Dans ses Mémoires, Madame de Genlis rapporte qu'une dame de sa connaissance allait jusqu'à se faire saigner pour conserver le teint pâle.

3. Pline l'Ancien explique comment fabriquer de la céruse. Il faut mettre du plomb dans des pots de vinaigre qu'on laisse bouchés dix jours, puis racler la « moisissure » formée, la broyer, la tamiser, la chauffer, la laver à l'eau douce, la faire sécher et, enfin, en faire des pastilles. Nous avons pu constater que cette méthode continue à être utilisée au cours des Temps Modernes. Dans l'Encyclopédie, on retrouve bel et bien la recette de Pline : le plomb réduit en lamelles est trempé dans du vinaigre, et il s'agit ensuite de racler la « rouille formée répétant cette opération jusqu'à ce que le plomb ait disparu ». La recette est parfois améliorée. Lémery (Dictionnaire universel...) et une seconde méthode proposée par l'Encyclopédie précisent que le plomb est étendu en lamelles minces, roulé autour de petits bâtons, puis déposé dans des pots de terre avec du vinaigre qu'on laisse dans le fumier pendant dix jours. Cette méthode a deux avantages : d'abord, le fumier optimise la réaction chimique; surtout, comme le plomb se présente sous la forme de spirales, la surface exposée à la vapeur du vinaigre est plus étendue ce qui permet de produire davantage de céruse en une seule fois.

4. Anne-Françoise Garçon (1998: 16) écrit : « Voici [...] le cas des fabriques de blanc de céruse, encore appelé blanc de plomb, une production qui manquait au royaume ». 5. D'après le marchand épicier et droguiste Pierre Pomet (1694), repris par Nicolas Lémery (1748).

6. André Le Fournier (1541); Michel Nostradamus (1572); Jean Liébaut (1582); Mademoiselle Meurdrac (1666, 1674); Nicolas de Blégny (1689); Simon Barbe (1689); Le 
Chevalier Digby (1689); Lémery (1695); Bruzen de la Martinière (1759); Pierre Joseph Buchoz (1771); Pons Augustin Alletz (1776); Dejean (1777).

7. Il en est de ces préparations comme des médicaments « couverts d'adjectifs » ou des substances aux « attributs les plus divers » évoqués par Gaston Bachelard (1938:112).

8. Selon Pierre Pomet (1694), les céruses de Hollande et d'Angleterre lui sont très inférieures car elle sont coupées de craies.

9. Selon l'expression d'Anne-Françoise Garçon (1998).

10. En fait, le mot céruse vient du latin cerussa, emprunté au grec keroussa, lui-même apparenté au terme keros, la cire.

11. C'est ici le même état d'esprit que celui qui préside à la fabrication des thériaques : « dans une thériaque qui réunit 150 substances, on ne s'occupe pas des proportions; on se confie à l'efficacité de la seule présence des ingrédients » (Bachelard 1938 : 113). Précisons que la thériaque est un remède prétendument universel, inventé par le médecin de Néron vers 40 après J.-C. et que l'on trouvait encore inscrit au Codex en 1884 ! Sur la pharmacopée de la fin du Moyen Âge et du début des Temps Modernes, on consultera avec profit la thèse de Jean-Pierre Bénézet (1999); on peut voir aussi Loïc Girre (1997).

12. Nous avons, en effet, trouvé quelques préparations de mouchoirs que l'on met à tremper plusieurs jours de suite dans des décoctions de céruse : dans ce cas, la quantité de céruse peut atteindre une livre.

13. Une fois encore, les analyses de Bachelard (1938: 113) peuvent s'appliquer à notre objet : la pharmacopée ancienne valorise « les mélanges les plus composés » et « la thériaque est une somme de substances jamais trop accueillante ».

14. On verra sur ce point les analyses de Jean-Pierre Goubert (1986).

15. Dans L'assemblée des femmes d'Aristophane, un jeune homme s'exclame « [...] mais qu'est-ce que peut bien être, je vous prie cette créature-là ? Une guenon toute enduite de céruse, ou une vieille ressuscitée de chez les morts ». Dans la Mostellaria, Plaute décrit la toilette de Philématie : la servante Scapha refuse de donner le rouge à sa maîtresse et s'en explique : « [...] à ton âge est-ce qu'on doit seulement toucher à aucune espèce de fard, blanc de céruse, blanc de mélos ou tout autre badigeonnage ». Et Martial d'écrire « [...] c'est ainsi que, plus noire que la mûre prête à tomber, Lycoris s'admire sous le fard de la céruse [...] » (Epigrammes I, 72).

16. Régnier, Satires, IX; Boileau, Epître, IX, cités par le Littré, art. « céruse ».

17. André Le Fournier, Michel Nostradamus, Jean Liébaut, Nicolas de Blégny, Nicolas Lémery, Pierre-Joseph Buchoz.

18. On verra, par exemple, l'ouvrage de Michel Nostradamus (1572) pour la fabrication de préparations blanchissantes au mercure et celui de Mademoiselle Meurdrac (1674) pour leur condamnation.

19. On trouve aussi ce principe parmi les trois principes magiques fondamentaux dégagés par Marcel Mauss (1902-1903) : c'est la « loi de similarité » selon laquelle « le semblable agit sur le semblable ».

20. En l'état actuel de notre recherche, nous pensons que durant le XVIe siècle et une grande partie du XVIIe siècle, les cosmétiques, fards et pommades de toutes sortes ont été souvent fabriqués à domicile par les usagers eux-mêmes ou par les valets et/ou les femmes de chambre. Plusieurs éléments permettent de le croire. D’abord, certains auteurs de manuels de cosmétique s'adressent à un public clairement identifié. Mademoiselle Meurdrac, par exemple, destine son ouvrage aux dames et ajoute dans l'avant-propos qu'elle souhaite « donner le moyen de fabriquer des remèdes sans 
dépenses » : la fabrication de ces recettes s'inscrit bien dans le cadre d'une économie domestique. Plus loin, après avoir décrit les fourneaux nécessaires aux opérations de chimie, elle explique qu'ils ne sont pas indispensables et que «l'on peut faire toutes les opérations sur un trépied entouré de briques, ou sur un réchaud, ou au coin de la cheminée... ». Simon Barbe adresse, d'abord, son livre aux « personnes de qualité » puis « aux gantiers, parfumeurs... ». Ensuite, le passage entre la profession de valet de chambre et celle de fabricant de cosmétiques existe : en 1539, est dressé l'inventaire après décès de François d'Escobart, valet de chambre et parfumeur du Roi (Archives Nationales, Minutier central, Étude LXXXVI/89). Le sieur Charles Decaux, parfumeur, était un ancien valet de chambre (Inventaire après décès de son épouse Marie Uzé, le 31 mai 1741, Archives Nationales, Minutier Central, Étude XXVIII/269). C'est sans doute au cours du XVIIIe siècle, afin de profiter d'un marché en pleine expansion, que la corporation des gantiers-parfumeurs a tenté de se saisir complètement de la production des cosmétiques. Et l'on en tient pour preuve, parmi d'autres, le fait que les manuels de la fin du siècle des Lumières, à l'inverse des précédents, s'adressent d'abord aux professionnels : Buchoz pense que son ouvrage peut être utile aux " parfumeurs, aux baigneurs-étuvistes et aux personnes chargées de la direction des toilettes ", Dejean écrit avoir conçu son ouvrage « pour les parfumeurs mais aussi pour les particuliers ».

21. À ce jour, nous avons dépouillé environ soixante-quinze inventaires après décès de parfumeurs ou d'épouses de parfumeurs (Minutier Central des Archives Nationales), soixante-neuf dossiers de faillites de parfumeurs et autres fabricants de cosmétiques (séries $\mathrm{D}^{4} \mathrm{~B} 6$ et $\mathrm{D}^{11} \mathrm{U} 3$ des Archives de la Seine), trente registres de commerce de ces professionnels (série $\mathrm{D}^{5} \mathrm{~B} 6$ des Archives de la Seine).

22. Selon Lémery (1748), ils contiennent de nombreuses drogues parmi lesquelles de la litharge, qui est un protoxyde de plomb.

23. D'après l'Encyclopédie, le blanc de perles n'est autre que du blanc de bismuth. On s'en servait comme d'un fard pour cacher les difformités du visage et l'on prétendait qu'il blanchissait le teint.

24.Archives Nationales, Minutier Central, Étude LXXXII/226.

25.Archives Nationales, Minutier Central, Étude XCIV/340.

26. Si le blanc en pain signalé dans l'inventaire après décès de Philippe Deldeuil est bien de la céruse, ce produit coûte, d'après l'estimation du notaire, vingt livres la livre. L'alun calciné coûtait quant à lui, au milieu du XVIIIe siècle, une livre la livre !

27. Lettre à Monsieur ${ }^{* * *}$ sur plusieurs maladies des yeux causées par l'usage du rouge et $d u$ blanc. Paris, 1760.

28. Malgré leurs dires, la voie cutanée n'est probablement pas la voie principale de pénétration de la céruse : l'épiderme sur lequel étaient appliquées les préparations protège le derme de manière efficace (même si une pénétration du plomb peut avoir lieu au niveau du bulbe des poils), et nulle pénétration dans le sang ne semble possible car les vaisseaux s'arrêtent au niveau du derme. En revanche, il est probable que de la poussière de céruse pouvait être inhalée ou/et être ingérée et ainsi, finir par passer dans le sang.

29. Archives de la Société Royale de Médecine, fonds des Remèdes Secrets, carton 97, dossier n 30 (Dame Colson, 1777); carton 100, dossier n 28 (Demoiselle Guérin, 1782). 30. Archives de la Société Royale de Médecine, fonds des Remèdes Secrets, carton 101, dossier n 37 (Sieur De la Houssaie, 1778). 
31. Archives de la Société Royale de Médecine, fonds des Remèdes Secrets, carton 105, dossier $n^{\circ} 24$ (Sieur De Viserant, 1788).

32. C'est ce que réclame la corporation des parfumeurs après la saisie effectuée sur les produits de la Demoiselle Martin (Archives de la Seine, série D4Z/3, Requête de

Mademoiselle Martin, marchande de rouge, présentée au Lieutenant Général de police, au sujet d'une saisie faite sur elle par les parfumeurs, 1778, et Réponse de la communauté des maîtres gantiers-boursiers-ceinturiers, de la ville de Paris prise lors d'une délibération du 6 août 1778). L'inquiétude des parfumeurs est particulièrement forte à cette date : la disparition puis le rétablissement des communautés (février et août 1776) ont créé une situation nouvelle. Dans un premier temps, la disparition des corporations rend le métier de parfumeur libre : il faut désormais faire une déclaration au Lieutenant Général de police pour avoir le droit d'exercer la profession. Les nombreuses recettes cosmétiques qui sont envoyées à la Société Royale de médecine à partir de 1776 afin d'obtenir une approbation témoignent bien de la liberté nouvelle d'entreprendre en ce domaine. L'édit d'août rétablit les corporations mais la situation reste floue : d'une part, les nouveaux statuts et règlements se font attendre, et d'autre part, il semble, à la lecture des jugements portés par la Société Royale de Médecine sur ces cosmétiques, que même après la Restauration, la liberté de ce commerce perdure de fait. Enfin, en partie peut-être à la faveur de ces réformes, un fait est porté au grand jour : la fabrication des fards, blanc et rouge, n'a jamais été réservée aux parfumeurs de manière explicite. En effet, d'après les sources dont nous disposons à ce jour (Statuts de la communauté des marchands gantiers-poudriers-parfumeurs, Édits, Arrêts du Parlement, Sentences de Police...), il n'est jamais clairement question de ces produits mais toujours de « choses dépendant du métier » ou « d'autres marchandises de parfums dépendant dudit état des maîtres gantiers-parfumeurs ». La Demoiselle Martin peut donc, à juste titre, évoquer « la liberté de notoriété publique » dont jouissait le rouge. Et, paradoxalement, la communauté des gantiers-boursiers-ceinturiers, contreattaquant la requête de la Demoiselle, ne dit pas autre chose quand, après avoir écrit : "Jamais la vente du rouge et du blanc n'ont été libres, pas plus que les autres marchandises de parfumerie », elle précise encore : «[...] les anciens statuts ne faisaient aucune distinction entre les marchandises [...] ». L'absence de toute mention spécifique du rouge ou du blanc pouvait tout aussi bien faire apparaitre leur fabrication comme libre ou non. À l'heure où nous relisons ces notes, il nous faut signaler l'ouvrage de Steven L. Kaplan (2001) dont la lecture attentive ne manquera pas de nous aider sur toutes ces questions.

33. Archives de la Société Royale de Médecine, fonds des Remèdes Secrets, carton 97, dossier n ${ }^{\circ} 61$ (Demoiselle De Clavigny, 1783) et carton 101, dossier nº30 (Sieur Lafaye 1780).

34. Archives de la Société Royale de Médecine, fonds des Remèdes Secrets, carton 101, dossier $\mathrm{n}^{\circ} 30$ (Sieur Lafaye, 1780).

35. Voir à ce sujet l'« Enquête épidémiologique sur l'utilisation cosmétique de produits dépigmentants par les femmes de Bamako (Mali) » dans les Annales de dermatologie et de vénéréologie, 1993, ainsi que les «Complications dermatologiques de l'utilisation de produits dépigmentants à Bamako (Mali) » dans les Annales de dermatologie et de vénéréologie, 1994. Ces deux articles ont été portés à notre connaissance par le Docteur Irina Bournérias, dermatologue. Qu'elle trouve ici l'expression de notre gratitude. 


\section{RÉSUMÉS}

Sous l'Ancien Régime, la blancheur du visage, symbole de la distinction, s'est imposée successivement à plusieurs catégories de la population. Destinés à permettre une fabrication domestique de cosmétiques, certains manuels de recettes accordent une place prépondérante aux préparations blanchissantes; la céruse est utilisée comme pigment blanc pour fabriquer des fards, mais aussi pour sa qualité détersive afin de faire disparaître les irrégularités de la peau. $\mathrm{Au}$ début du XVIIIe siècle, la corporation des gantiers-parfumeurs cherche à étendre ses prérogatives professionnelles à la fabrication des cosmétiques mais leur utilisation de la céruse s'avère très limitée : elle coûte cher et ils en connaissent le caractère toxique. En effet, la médecine des Lumières dénonce vivement la nocuité des préparations cosmétiques, en particulier celle des blancs de plomb, et les inventeurs, simples particuliers ou professionnels, invoquent désormais l'argument que leur cosmétique ne nuit pas à la santé du consommateur.

\section{The use of white-lead in cosmetics during the old Regime (16th-18th centuries)}

During the Old Regime, the paleness of the complexion - sign of social distinction- imposed itself to several categories of the population and some do-it-yourself cosmetics handbooks even focussed on whitening preparations. Ceruse was used as a white pigment for make-up but also as a cleansing agent to rub out the imperfections of the skin. At the turn of the 18th century, the guild of glover-perfumers tried to expand its professional prerogatives to the making of cosmetics. However, the use of white-lead did not widespread because of its price and its toxicity. The noxiousness of cosmetics - especially those including ceruse- was vividly denounced by the physicians of Enlightenment. From then on, harmlessness was put forth by the producers as a sales argument for their preparations.

El uso de la cerusa para la fabricación de cosméticos durante el Antiguo Régimen (s. XVIXVIII)

Durante el Antiguo Régimen, la blancura del semblante, símbolo de distinción, se impuso sucesivamente a varias categorías sociales. En vista de la fabricación doméstica de cosméticos, algunos libros de recetas otorgan un lugar preponderante a las preparaciones blanqueadoras. La cerusa se utiliza como pigmento blanco para fabricar afeites y maquillajes; además, sus propiedades detergentes sirven para eliminar las desigualdades de la piel. A principios del siglo XVIII, el gremio de los guanteros-perfumeros trata de extender el campo de sus prerrogativas profesionales hasta incluir la fabricación de cosméticos, pero su empleo de la cerusa resulta ser muy limitado : es cara, y conocen la toxicidad de esta sustancia. De hecho, la medicina del siglo de las Luces denuncia nítidamente la nocividad de las preparaciones cosméticas, en espacial la de los blancos demomo, y los inventores, simples particulres o profesionales, de aquí en adelante, deberán argüir que su cosmético no perjudica a la salud del consumidor.

\section{INDEX}

Mots-clés : céruse, corporations, cosmétique, médecine, parfumerie 
AUTEUR

\section{CATHERINE LANOË}

Ater au Collège de France, chercheur associé à l'Institut d'Histoire Moderne et Contemporaine (IHMC), 45 rue d'Ulm, 75005 Paris 